Article

\title{
Comparisons of Scotopic/Photopic Ratios Using 2- and 10-Degree Spectral Sensitivity Curves
}

\author{
Jianhua Ding ${ }^{1}$, Qi Yao ${ }^{1,2, *(1)}$ and Lei Jiang ${ }^{3}$ \\ 1 College of Architecture and Urban Planning, Shenzhen University, Shenzhen 518060, China; a-djh@163.com \\ 2 Beijing Advanced Innovation Centre for Future Urban Design, Beijing University of Civil Engineering and \\ Architecture, Beijing 102627, China \\ 3 No. 9, 518 Lane, Ji'nian East Rd, Baoshan, Shanghai 200439, China; leojianglei@163.com \\ * Correspondence: yaoqi@szu.edu.cn; Tel.: +86-1352-875-6877
}

Received: 12 September 2019; Accepted: 15 October 2019; Published: 22 October 2019

\begin{abstract}
Despite the fact that a 2-degree spectral sensitivity curve (SSC) is extensively used in scientific research and relevant applications, the choice between the 10-degree or the 2-degree photopic SSCs in practical applications for the calculation of scotopic/photopic ratios ( $S / P$ ratios) depends on actual needs. We examined $S / P$ ratios for more than 300 light sources for correlated colour temperatures (CCTs) from $2000 \mathrm{~K}$ to $8000 \mathrm{~K}$ and blackbody radiant spectra from $10000 \mathrm{~K}$ to $45000 \mathrm{~K}$ using 2- and 10-degree SSCs. Results showed that the ratio of the $S / P$ values calculated using the 10-degree and 2-degree SSCs was approximately equal to 0.916 . The average mesopic luminance difference increased from $0 \%$ to $5.7 \%$ at a photopic adaptation luminance from 0.005 to $5 \mathrm{~cd} / \mathrm{m}^{2}$. For most practical applications, the mesopic luminance values calculated using these two SSCs were different by several percentage units, yet these differences could be neglected. At extremely high CCTs over $10000 \mathrm{~K}$, the mesopic luminance difference may approximate the maximum value of $16 \%$. This work proposes the conversion coefficients for $S / P$ ratios and the transforming mesopic luminance values calculated for 2- and 10-degree SSC systems. These results may help researchers clarify differences between the $S / P$ ratios calculated using different SSCs.
\end{abstract}

Keywords: mesopic vision; luminous radiation efficacy; scotopic vision; mesopic luminance; photopic adaptation luminance

\section{Introduction}

There are three vision states for human beings, photopic vision with luminance values $>5 \mathrm{~cd} / \mathrm{m}^{2}$, mesopic vision with luminance values from 0.005 to $5 \mathrm{~cd} / \mathrm{m}^{2}$, and scotopic vision with luminance values $<0.005 \mathrm{~cd} / \mathrm{m}^{2}$ [1]. The luminance of values of several scenes, like roads, tunnels, and dashboard lightings, likely fall in the mesopic vision range. To measure light source performances in mesopic vision states, the scotopic/photopic ratio ( $S / P$ ratio) is used [2]. Effectively, this is the ratio of the luminous efficacy of radiation (LER) in scotopic and photopic vision. There are two photopic spectral sensitivity curves (SSCs): one is the Commission Internationale de 1'Eclairage (CIE) 1924 2-degree standard observer SSC [3] and the other is the CIE 1964 10-degree standard observer SSC [4]. Some studies claim that the 10-degree SSC constitutes a better representation [5]. Prior research studies on colour differences also show that 10-degree colour matching functions yield smaller colour differences than 2-degree colour matching functions [6]. Another study [7] suggested that there may be problems in the short-wavelength part of the 2-degree SSC. In road lighting, visual tasks sometimes require large visual fields, while peripheral vision is also important [8-10]. The rod photoreceptors are mainly distributed in the part of the retina associated with peripheral vision. We may thus conclude that both the 10- and 2-degree systems should be considered for uses in various applications [11]. 
CIE recommended mesopic vision models for road lighting [2] and published guidelines on how to use the model [12]. However, CIE did not recommend which SSC should be adopted for calculation, implying that either SSC type can be used in applications. In most of the published research studies and practical applications, the 2-degree SSC system has been used. Berman [13] reported S/P ratios of 16 typical light sources in the range from 0.23 to 2.47. Lita et al. [14] reported a light source with an $\mathrm{S} / \mathrm{P}$ ratio of 2.56 at a correlated colour temperature (CCT) of $5368 \mathrm{~K}$. Nizamoglu et al. [15] reported a white-light emitting diode (WLED) with an S/P ratio of 3.04 at the extremely high CCT of $45000 \mathrm{~K}$. Guo et al. [16] proposed light-emitting diodes (LEDs) for CCTs from $2700 \mathrm{~K}$ to $45000 \mathrm{~K}$ with S/P ratios in the range from 1.83 to 3.84. Zan et al. [17] reported WLEDs that reached S/P ratios from 1.54 to 3.84, which corresponded to CCTs in the range from $2700 \mathrm{~K}$ to $7580 \mathrm{~K}$. Li et al. [18] reported two-hump quantum-dot-based LEDs that reached an $S / P$ ratio value of 2.09 at $4038 \mathrm{~K}$, and a four-hump LED that reached an $S / P$ ratio value of 3.08 at $5015 \mathrm{~K}$. Zhang et al. [19] optimised hybrid white LEDs for high $S / P$ ratios at varying CCTs that reached high colour rendition, and compared their differences. Despite the fact that in some studies it has not been mentioned which SSC was used, we rechecked the spectra in the literature and have confirmed that most studies have used 2-degree SSCs. To date, no study has compared the differences of the calculations of $S / P$ ratios using these two SSCs. In practical applications, there are urgent needs for large-scale assessments of urban lighting [20] and nightscape lighting. However, there are some unsolved technical issues to assure photopic adaptation luminance [21,22], which may cause inaccuracy in assessments.

In this study, we examined the differences of the calculated $S / P$ ratios with two SSCs based on tests with more than 300 light sources [23] with CCTs from $2000 \mathrm{~K}$ to $8000 \mathrm{~K}$, and with blackbody radiant (BR) light sources with CCTs from $10000 \mathrm{~K}$ to $45000 \mathrm{~K}$. We calculated the mesopic luminance levels at photopic adaptation luminance levels from 0.005 to $5 \mathrm{~cd} / \mathrm{m}^{2}$ based on the use of the two tested SSCs and compared the differences. Conversion coefficients between the S/P ratio and mesopic luminance calculated using 2- and 10-degree SSCs were proposed. The work may help elucidate the S/P ratios and mesopic luminance differences calculated with these two different SSCs in research studies, and provide convenient conversions in practical applications.

\section{S/P Ratio of Monochromatic Spectra}

The $S / P$ ratio was calculated by dividing the LER in scotopic vision $\left(\mathrm{LER}_{\mathrm{S}}\right)$ by that in photopic vision $\left(\mathrm{LER}_{\mathrm{p}}\right) . \mathrm{LER}_{\mathrm{p}}$ was defined by Equation (1), LER $\mathrm{s}$ was defined by Equation (2), and the $S / P$ ratio, which can also be expressed as a quotient in the form of Rsp [12], was defined with the use of Equation (3). Herein, $P(\lambda)$ represents the spectral power distribution (SPD) of the light source, $K_{m}$ is the maximum photopic luminous efficacy of $683 \mathrm{~lm} / \mathrm{W}$ at $555 \mathrm{~nm}$, and $K_{m}$ is the maximum scotopic luminous efficacy of $1700 \mathrm{~lm} / \mathrm{W}$ at $507 \mathrm{~nm}$. Accordingly, $V(\lambda)$ is the SSC for photopic vision. Therefore, in the calculations, we used $V_{2}(\lambda)$ to denote the 2-degree and $V_{10}(\lambda)$ to denote the 10-degree systems, respectively. $V^{\prime}(\lambda)$ is the SSC for scotopic vision. Both $V(\lambda)$ and $V^{\prime}(\lambda)$ are shown in Figure 1.

To compare the $S / P$ ratio differences at each wavelength, we calculated the $S / P$ ratios of monochromatic wavelengths for a full width at half maximum (FWHM) distance of $1 \mathrm{~nm}$, from 380 to $780 \mathrm{~nm}$, as shown in Figure 2. The $S / P$ ratio of an equal energy white (EEW) light calculated using 2-degree SSC is 2.261 and 10-degree is 2.071 , and the difference is approximately $9 \%$. It is clearly noted that the main differences appear in the short-wavelength range $(<500 \mathrm{~nm})$. 


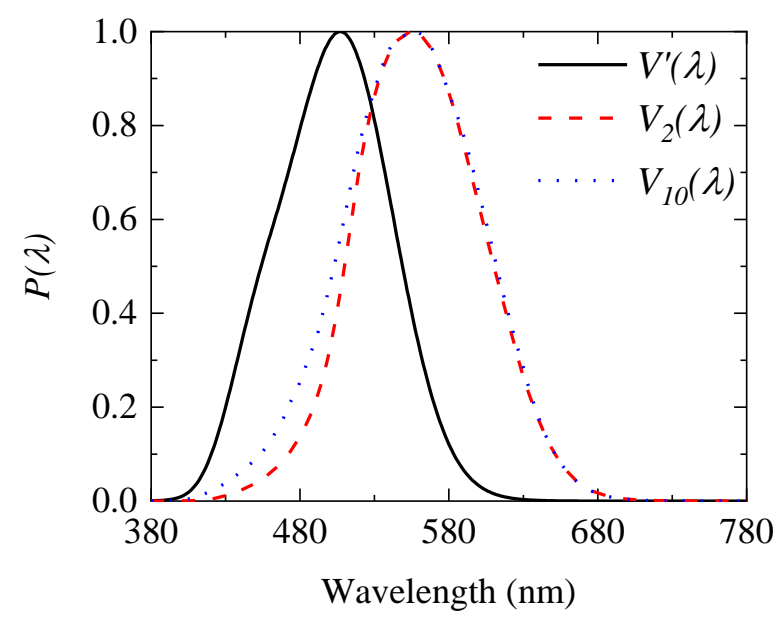

Figure 1. Spectral sensitivity curves (SSCs) for scotopic vision and photopic vision using 2- and 10-degree systems.
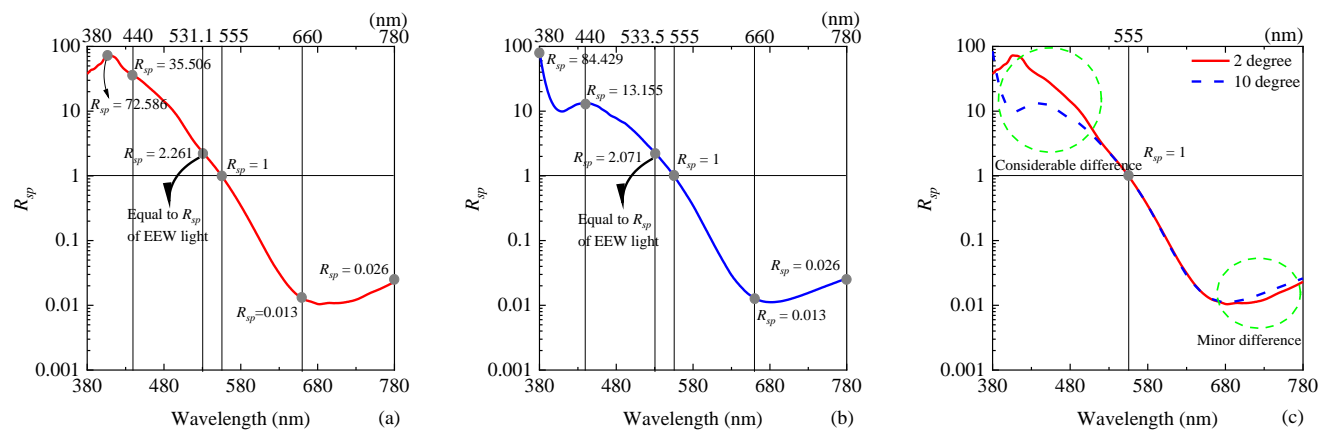

Figure 2. Scotopic/photopic ratios $\left(R_{s p}\right)$ as a function of wavelength using (a) 2-degree and (b) 10-degree SSCs. Some special $R_{s p}$ values have been marked. The $R_{s p}$ at $555 \mathrm{~nm}$ is equal to unity. The $R_{s p}$ values at 531.1/533.5 $\mathrm{nm}$ are 2.261/2.085, and are equal to those for equal energy white (EEW) light in the 2-degree/10-degree cases. (c) Comparison of $S / P$ ratios using 2- and 10-degree SSCs. There is a considerable difference in the short-wavelength part, especially for values $<500 \mathrm{~nm}$, and a minor difference in the long wavelength part.

$$
\begin{gathered}
L E R_{\mathrm{p}}=\frac{K_{m} \int_{380}^{780} P(\lambda) V(\lambda) d \lambda}{\int_{380}^{780} P(\lambda) d \lambda} \\
L E R_{\mathrm{s}}=\frac{K_{m}^{\prime} \int_{380}^{780} P(\lambda) V^{\prime}(\lambda) d \lambda}{\int_{380}^{780} P(\lambda) d \lambda} \\
S / P \text { ratio }=\frac{K_{m}^{\prime} \int_{380}^{780} P(\lambda) V^{\prime}(\lambda) d \lambda}{K_{m} \int_{380}^{780} P(\lambda) V(\lambda) d \lambda} .
\end{gathered}
$$

\section{Relation of S/P Ratios of Light Sources Using 2-degree and 10-degree SSCs}

Monochromatic spectra calculated for the $S / P$ ratios in Section 2 are considered extreme conditions. In this section, we investigated a set that contained more than 300 light sources. Most of these were commercial light sources. These light source data were presented in the Illuminating Engineering Society of North America (IES-30-15. 2015), including incandescent lamps, fluorescent lamps, high-intensity discharge lamps, and LEDs. The CCTs of these light sources ranged from 2000 to $8000 \mathrm{~K}$. The SPDs were normalised to a maximum intensity of unity. The mean SPD is shown in Figure 3. We calculated 
$S / P$ ratios of these light sources using both 2- and 10-degree SSCs. Figure 4 shows that the $S / P$ ratio was calculated in the plot of the 10-degree against the 2-degree SSC system. The relation is fitted well by a linear function with a high coefficient of determination, $R^{2}=0.999$. The slope of the linear function is approximately equal to the ratio of the EEW light, which is equal to 2.071/2.261 0.916. We can use 0.916 as the conversion coefficient for the transformation of the results calculated with the use of the 2-and the 10-degree SSCs.

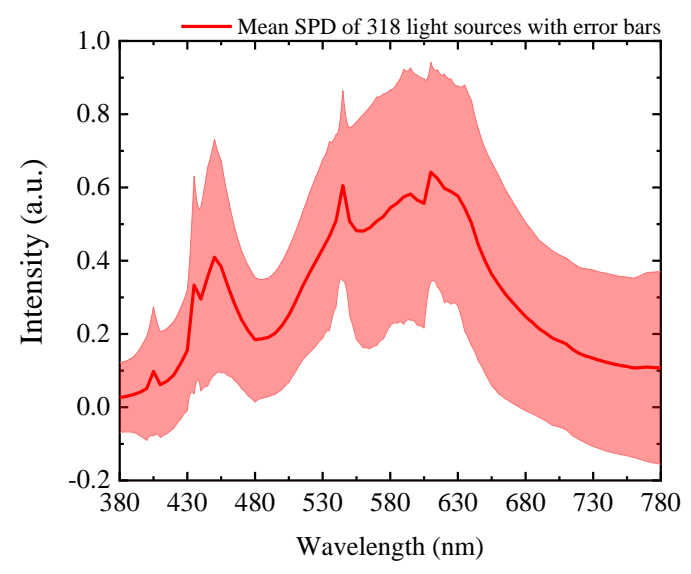

Figure 3. Mean spectral power distribution (SPD) of 318 light sources with error bars, as provided by the Illuminating Engineering Society of North America (IES-30-15). The corresponding colorimetric parameters were as follows: correlated colour temperatures (CCT) $3628 \mathrm{~K}, \mathrm{x}=0.397, \mathrm{y}=0.384$, CIE Ra $=87, \mathrm{IES} \mathrm{R}_{\mathrm{f}}=86$, and $\mathrm{R}_{\mathrm{g}}=100$.

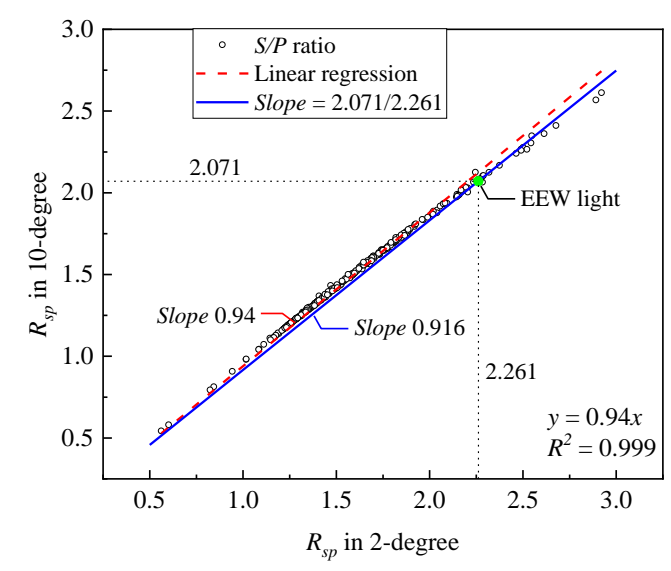

Figure 4. $R_{s p}$ calculated in a plot of the 10-degree against the 2-degree SSC system. The red-dashed line is a linear regression fitting with a slope of 0.94 . The blue solid line is a straight line with a slope of 0.916 , which is equal to the ratio of the $R_{s p}$ values for EEW light in the 10-degree (2.071) and 2-degree (2.261) systems.

Due to the fact that there are no differences in the responses for scotopic vision, the $S / P$ ratio difference is caused by the difference of $L R_{p}$. Although considerable differences may exist for $S / P$ ratios, the differences for mesopic luminance values may not be as considerable. We should further examine the mesopic luminance values transformed from photopic adaptation luminance levels in mesopic vision states.

\section{Mesopic Luminance Values at Different Photopic Adaptation Luminance Levels}

Figure 5 shows the plot of the 10-degree versus the 2-degree luminance values at the photopic adaptation luminance levels of $0.01,0.03,0.1,0.3,1$, and $3 \mathrm{~cd} / \mathrm{m}^{2}$. These data are well fitted (high 
$\mathrm{R}^{2}$ values) with linear functions. This implies that photopic adaptation luminance is the main factor that influences the results. The slope increases from 1.01 to 2.23 with photopic adaptation luminance values in the range from 0.01 to $3 \mathrm{~cd} / \mathrm{m}^{2}$. For photopic adaptation luminance values $<0.1 \mathrm{~cd} / \mathrm{m}^{2}$, the differences are very small. When the luminance values $>0.3 \mathrm{~cd} / \mathrm{m}^{2}$, the plotted points are scattered.

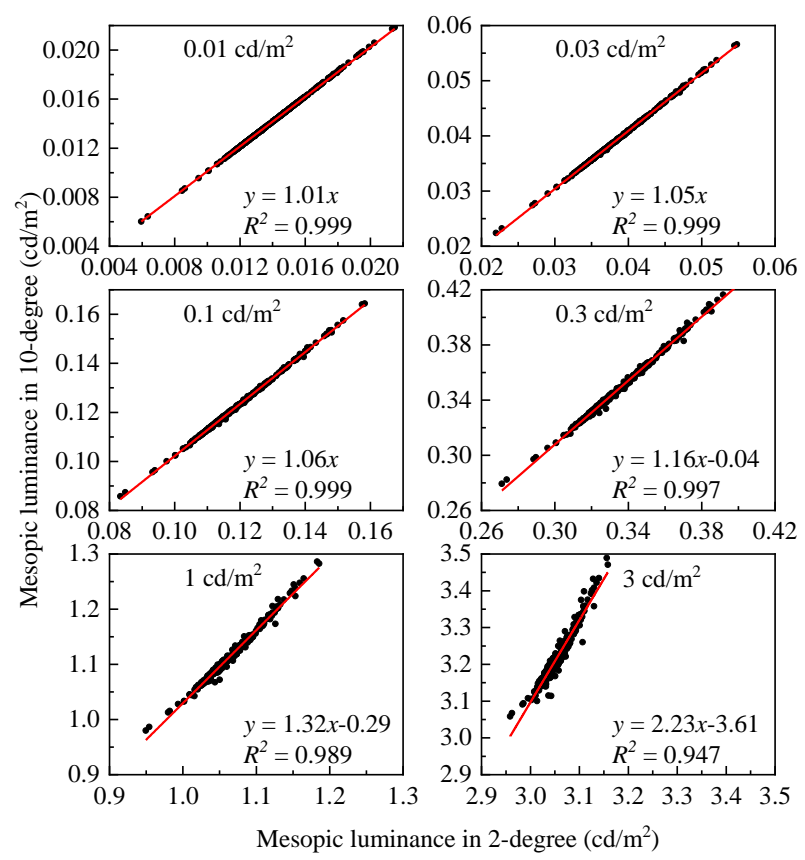

Figure 5. Mesopic luminance plots of the 10- against the 2-degree systems at the reference photopic adaptation luminance levels of $0.01,0.03,0.1,0.3,1$, and $3 \mathrm{~cd} / \mathrm{m}^{2}$. The reference photopic adaptation luminance level is included in the 2-degree SSC system. Accordingly, corresponding photopic adaptation luminance values in the 2-degree SSC system should be multiplied with the conversion coefficient $R_{\mathrm{sp}}$ (2-degree)/ $R_{\mathrm{sp}}$ (10-degree).

Figure 6 shows the mesopic luminance ratio estimated by dividing the mesopic luminance values of the 10-degree SSC by the 2-degree SSC. The mesopic luminance ratio increases as the photopic adaptation luminance increases from 1.000 in scotopic vision to 1.057 in photopic vision. Specifically, the difference of mesopic luminance ranged from $0 \%$ to $5.7 \%$ based on the calculations with the use of the two SSCs.

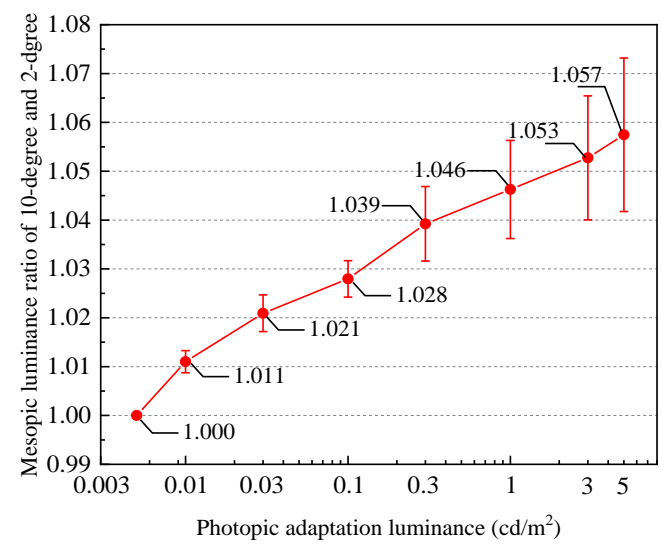

Figure 6. Mesopic luminance ratio using 10- and 2-degree light source SSCs at photopic adaptation luminance values from 0.005 to $5 \mathrm{~cd} / \mathrm{m}^{2}$. 


\section{Examinations of BR Light Sources at Extremely High CCTs}

At higher CCTs, the differences of the S/P ratio calculated using two 2-degree and 10-degree SSC systems tend to be greater. We invested BR light sources in the temperature range from 10000 to $45000 \mathrm{~K}$, and results are shown in Figure 7. The differences increase with CCT, and they are over $10 \%$. Figure 8 shows the mesopic luminance ratio as a function of CCT and the photopic adaptation luminance. The mesopic luminance ratio increased as the CCT and photopic adaptation luminance increased. The maximum difference was approximately equal to $16 \%$.

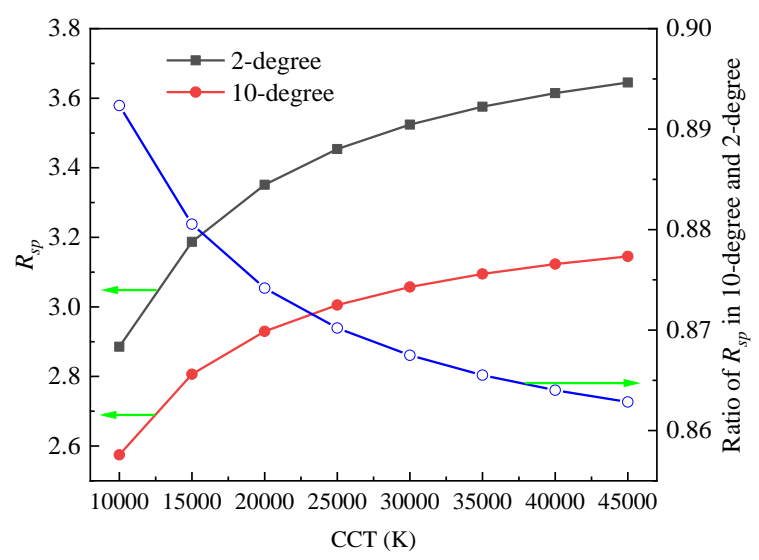

Figure 7. $R_{s p}$ variations for CCTs in the range from 10000 to $45000 \mathrm{~K}$ and ratio of $R_{s p s}$ in 10 - and 2-degree SSC systems as a function of CCT.

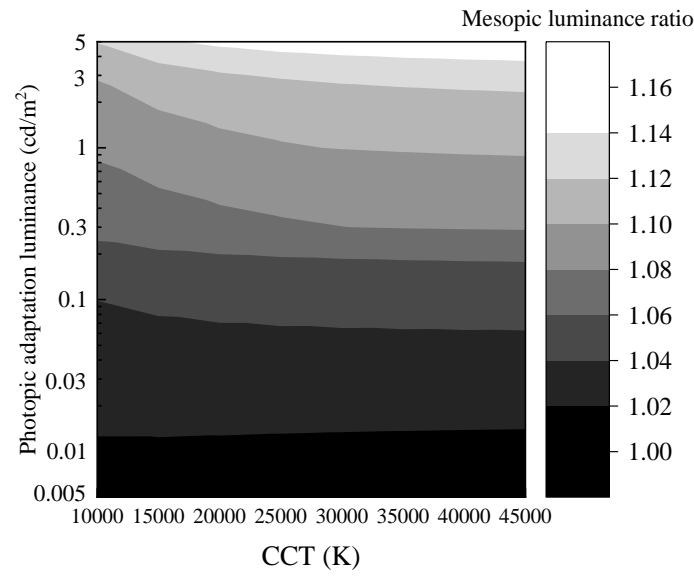

Figure 8. Mesopic luminance ratio using 10- and 2-degree SSCs as a function of CCT and photopic adaptation luminance.

\section{Examination of a Real Lighting Scene}

We measured luminance distribution of a road in Shenzhen using a typical phosphor converted LED type. The road is $140 \mathrm{~m}$ in length and $16 \mathrm{~m}$ in width, as shown in Figure 9. We used a spectral image radiance colorimeter EVERFINE SIRC-2000 to measure the luminance distributions and spectral information of light sources. The colorimeter was set at $60 \mathrm{~m}$ far away and $1.5 \mathrm{~m}$ in height in each measurement. The luminaire was measured at a CCT of $3650 \mathrm{~K}$, and the $S / P$ ratio in the 2- and 10-degree SSCs were 1.33 and 1.28, respectively. The average luminance was approximately $1 \mathrm{~cd} / \mathrm{m}^{2}$. The referred paradigm was a typical road lighting application at moderate CCT and luminance levels. We transformed the photopic adaptation luminance to mesopic luminance in a point-by-point manner. Figure 10 shows the luminance and the corrected mesopic luminance distributions. There are 810 luminance points in the road, which form the luminance distribution contours. The difference 
between the corrected mesopic luminance using both the 10- and 2-degree SSCs of these 810 points is approximately $3 \%$ on average, as shown in Figure 11.

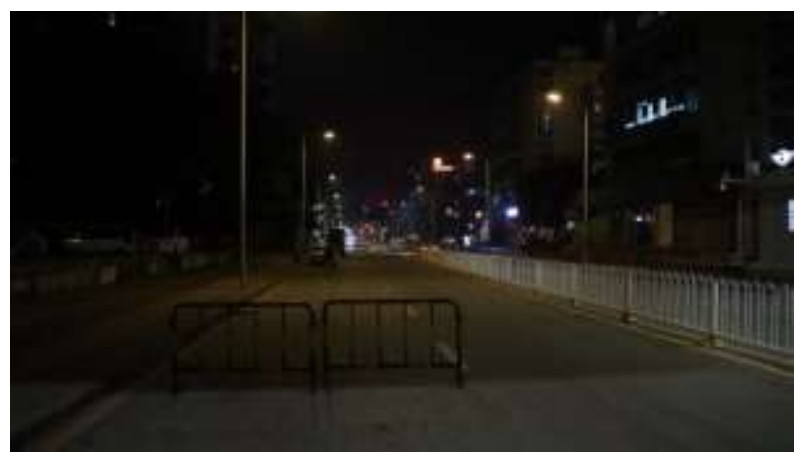

Figure 9. Measurements conducted in a typical road in Shenzhen with a length of $140 \mathrm{~m}$ and a width of $16 \mathrm{~m}$.
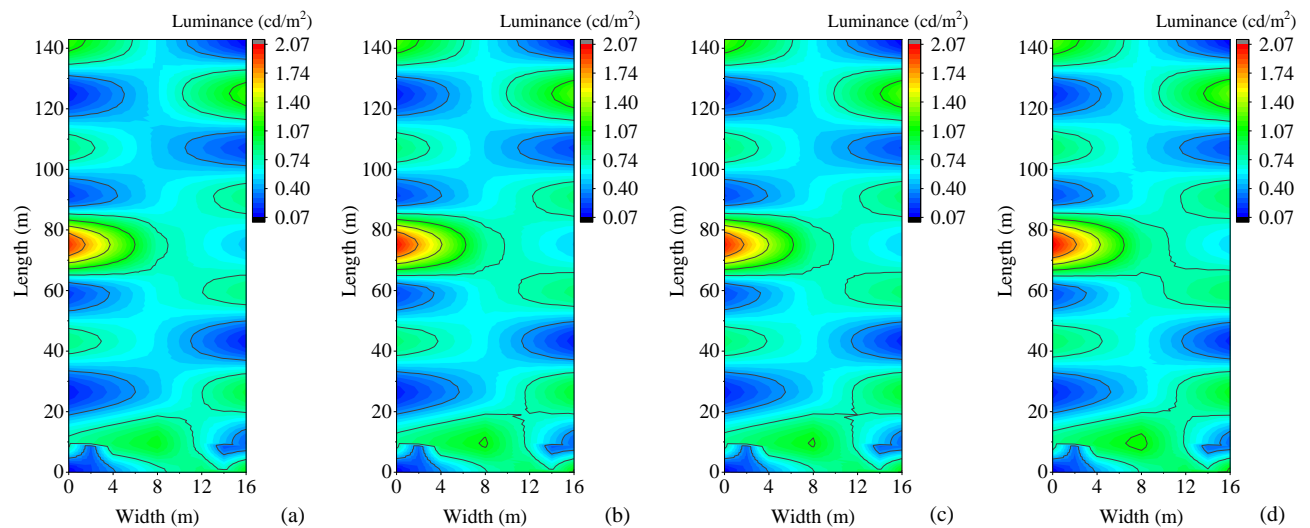

Figure 10. Luminance distributions and corrected mesopic luminance distributions. (a) Luminance distributions measured using the 2-degree SSC system, (b) conversion to luminance distributions using the 10-degree SSC system, (c) corrected mesopic luminance using the 2-degree system, and (d) corrected mesopic luminance using the 10-degree system.

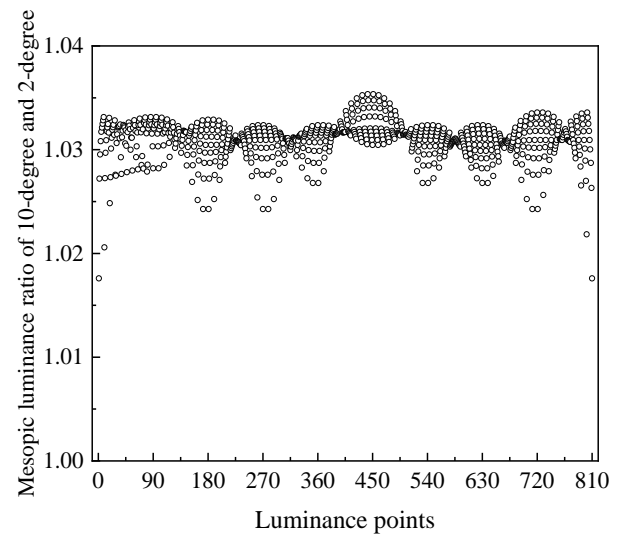

Figure 11. Mesopic luminance ratios of 10-degree and 2-degree SSCs of 810 luminance points. The mean ratio is 1.03 , which yields a difference of $\sim 3 \%$.

\section{Discussion and Conclusion}

In many applications, the 2-degree SSC was used for the calculation of the LER and S/P ratio. However, in some applications, a large visual field was required, and a 10-degree SSC was more 
reasonable. We analysed the difference between these two SSCs based on an investigation of more than 300 light sources and extremely high CCT BR sources.

Comparison of the $S / P$ ratios calculated using monochromatic light sources yielded considerable differences in the short-wavelength region $(<500 \mathrm{~nm})$. Light sources operating at high CCT values will likely cause considerable differences. In the very short-wavelength range $(<430 \mathrm{~nm})$, the S/P ratio increased abruptly. However, human eyes are not sensitive to changes in this region, and the precision of SSCs cannot guarantee the precision of the extremely high $S / P$ ratio.

In the mesopic vision range, the differences in the mesopic luminance values of the 300 light sources calculated with the two SSCs increased as the photopic adaptation luminance values increased. In the scotopic vision cases, the same SSC $V^{\prime}(\lambda)$ was applied and there were no differences. In photopic vision cases, the average difference reached a value of $\sim 6 \%$. For some practical road lighting applications with luminance values less than $1 \mathrm{~cd} / \mathrm{m}^{2}$ and with moderate CCTs at approximately $4000 \mathrm{~K}$, the difference of the corrected mesopic luminance values based on the two different SSCs studied herein was approximately $3 \%$. For high CCT values (>6000 K), light sources, and high luminance levels $\left(>1 \mathrm{~cd} / \mathrm{m}^{2}\right)$, the difference may reach $5 \%$.

Accordingly, as the differences of the two SSCs increase, the CCTs of the light sources also increased. The differences were relatively small and with values $<5 \%$ for light sources operating with CCTs in the range from 2000 to $8000 \mathrm{~K}$. For CCTs $>10000 \mathrm{~K}$, the error at some mesopic luminance levels reached values $>10 \%$. In some research work, $C C T$ light sources with extremely high $S / P$ ratios have been reported. The $S / P$ ratios for these light sources calculated using the two SSCs are so different that they require further analyses. For some special applications, like dashboard illumination in driving cabins and landscape lightings, blue LEDs might be used. Accordingly, considerable differences may be evoked. However, a few light sources applied in functional lighting operate at extremely high CCTs.

Generally, the average difference of the $S / P$ ratio is approximately $8 \%$ for light sources operating at CCTs in the range from 2000 to $8000 \mathrm{~K}$. The mesopic luminance values, which are related to both the photopic adaptation luminance and $C C T$, do not yield considerable $S / P$ ratio differences. For functional lighting, the photopic adaptation luminance is the main reason for the evoked differences.

In summary, several valuable inferences were drawn based on the findings of this study. Firstly, in most conditions, to transform the S/P ratio calculated using the 2- and 10-degree SSC outcomes, a conversion coefficient equal to 0.916 should be used. Secondly, in most practical applications, the difference of the calculated mesopic luminance using 2- and 10-degree SSCs was approximately in the range of 3\%-5\%, and the conversion coefficients ranged from 1.03 to 1.05 . Thirdly, for applications with CCTs $>10,000 \mathrm{~K}$, and for luminance values $>1 \mathrm{~cd} / \mathrm{m}^{2}$, the differences may be $>10 \%$, and mesopic luminance should be calculated using suitable SSCs.

Most urban lighting scenes, such as road lighting and tunnel lighting, are in the mesopic vision range, and in the nightscape lighting. Most of these lighting scenes require large visual field, while others may require small visual fields to observe. Applying the suitable SSC to reach more precise assessments could help improve the lighting design, yield a smaller energy consumption, and reduce the risk of potential light pollutions.

There are some limitations associated with this study. Firstly, it is difficult to determine a very precise photopic adaptation luminance for complex luminance distributions because adaptation luminance may be related to luminance distributions, eye movements, and surrounding luminance effects [21]. Secondly, the adaptation luminance determined from 2-degree and 10-degree visual fields should be different, because the visual angles and adaptation fields [12] are different. The CIE TN 007:2017 recommends the adaptation field to be taken as the design area, and the adaptation luminance as the average luminance of the adaptation field. This is not applicable to designing suitable adaptation fields for each observation point when assessing a large-scale urban lighting scene. There is no feasible way at present to apply a suitable adaptation luminance for the entire road. Therefore, in this work, we only consider the SSC as the factor influencing photopic adaptation luminance and adopt a local photopic adaptation luminance for each observation point. 
Author Contributions: Conceptualisation, Jianhua. Ding. and Qi. Yao.; methodology, Qi. Yao.; validation, Lei. Jiang.; formal analysis, Lei. Jiang.; investigation, Jianhua Ding.; writing_original draft preparation, Jianhua Ding. and Qi Yao.; writing—review and editing, Qi. Yao.; funding acquisition, Qi.Yao.

Funding: This research was funded by the National Natural Science Foundation of China (NSFC) (grant number 61605125) and Beijing Advanced Innovation Center for Future Urban Design, Beijing University of Civil Engineering and Architecture (Project No. UDC2018010411).

Conflicts of Interest: The authors declare no conflict of interest.

\section{References}

1. CIE 115:2010: Lighting of Roads for Motor and Pedestrian Traffic; CIE: Vienna, Austria, 2010.

2. CIE 191:2010: Recommended System for Mesopic Photometry based on Visual Performance; CIE: Vienna, Austria, 2010.

3. CIE. Commission Internationale de l'Eclairage Proceedings; Cambridge University Press: Cambridge, UK, 1926.

4. CIE Proceedings (1964) Vienna Session (Committee Report E-1.4.1); Bureau Central de la CIE: Paris, France, 1963; Volume B, pp. 209-220.

5. Stockman, A.; Sharpe, L.T. Human cone spectral sensitivities: a progress report. Vis. Res. 1998, 38, $3193-3206$. [CrossRef]

6. Shaw, M.Q. Evaluating the 1931 CIE color matching functions. In Proceedings of the AIC, The University of Hertfordshire, UK, 25-30 May 1997.

7. Sharpe, L.T.; Stockman, A.; Jagla, W.; Jagle, H. A luminous efficiency function, $\mathrm{v}^{*}(\mathrm{l})$, for daylight adaptation. J. Vis. 2005, 5, 948-968. [CrossRef] [PubMed]

8. Boyce, S.F.P.; Richards, M. Road lighting and energy saving. Lighting Res. and Technol. 2009, 41, $245-260$. [CrossRef]

9. Akashi, Y.; Rea, M.S. Peripheral detection while driving under a mesopic light level. J. Illuminating Eng. Soc. 2002, 31, 85-94. [CrossRef]

10. Akashi, Y.; Rea, M.S.; Bullough, J.D. Driver decision making in response to peripheral moving targets under mesopic light levels. Lighting Res. Technol. 2007, 39, 53-67. [CrossRef]

11. Stockman, A.; Sharpe, L.T. Into the twilight zone: the complexities of mesopic vision and luminous efficiency. Ophthalmic Phys. Optics 2006, 26, 225-239. [CrossRef] [PubMed]

12. CIE TN 007:2017: Interim Recommendation for Practical Application of the CIE System for Mesopic Photometry in Outdoor Lighting; CIE: Vienna, Austria, 2017.

13. Berman, S.W. Energy efficiency consequences of scotopic sensitivity. J. IES 1992, 1, 3-14. [CrossRef]

14. Lita, A.; Washington, A.L.; van de Burgt, L.; Strousse, G.F.; Stiegman, A.E. Stable efficient solid-state white-light-emitting phosphor with a high scotopic/photopic ratio fabricated from fused CdSe-silica nanocomposites. Adv. Mater. 2010, 22, 3987. [CrossRef] [PubMed]

15. Nizamoglu, S.; Erdem, T.; Demir, H. High scotopic/photopic ratio white-light-emitting diodes integrated with semiconductor nanophosphors of colloidal quantum dots. Opt. Lett. 2010, 36, 1893. [CrossRef] [PubMed]

16. Guo, Z.Q.; Shih, T.M.; Lu, Y.J.; Gao, Y.-L.; Zhu, L.-H.; Chen, G.-L.; Zhang, J.-H.; Lin, S.-Q.; Chen, Z. Studies of scotopic/photopic ratios for color-tunable white light-emitting diodes. IEEE Photon J. 2013, 5, 8200409.

17. Zan, L.L.; Lin, D.Y.; Zhong, P.; He, G. Optimal spectra of white led integrated with quantum dots for mesopic vision. Opt. Express 2016, 24(7), 7643. [CrossRef] [PubMed]

18. Li, F.; You, L.; Nie, C.; Zhang, X.J.; Li, H.; Gu, X.; Huang, Y.; Li, Q. Quantum dot white light emitting diodes with high scotopic/photopic ratios. Opt. Express 2017, 25(18), 21901. [CrossRef] [PubMed]

19. Zhang, C.W.; Xiao, L.C.; Zhong, P.; He, G. Photometric optimization and comparison of hybrid white LEDs for mesopic road lighting. Appl. Opt 2018, 57, 4665-4671. [CrossRef] [PubMed]

20. Yao, Q.; Wang, H.B.; Uttley, J.; Zhuang, X. Illuminance reconstruction of road lighting in urban areas for efficient and healthy lighting performance evaluation. Appl. Sci. 2018, 8, 1646. [CrossRef]

21. Uchida, T.; Ayama, M.; Akashi, Y.; Hara, N.; Kitano, T.; Kodaira, Y.; Sakai, K. Adaptation luminance simulation for CIE mesopic photometry system implementation. Lighting Res. Technol. 2016, 48, 14-25. [CrossRef] 
22. Gao, C.; Xu, Y.; Wang, Z.; Melosa, M.; Pointer, M.; Luo, M.R.; Xiao, K.; Li, C. Improved computation of the adaptation coefficient in the CIE system of mesopic photometry. Opt. Express 2017, 25, 18365. [CrossRef] [PubMed]

23. Illuminating engineering society. IES method for evaluating light source colour rendition. IES 2015, 15-30. 